\title{
A study of photometric variations on the dwarf planet (136199) Eris ${ }^{\star}$
}

\author{
R. Duffard ${ }^{1}$, J. L. Ortiz ${ }^{1}$, P. Santos Sanz ${ }^{1}$, A. Mora ${ }^{2}$, P. J. Gutiérrez ${ }^{1}$, N. Morales ${ }^{1}$, and D. Guirado ${ }^{1}$ \\ 1 Instituto de Astrofísica de Andalucía, CSIC, Apt 3004, 18008 Granada, Spain \\ e-mail: duffard@iaa.es \\ ${ }^{2}$ Universidad Autónoma de Madrid, Departamento de Física Teórica C-XI, 28049 Madrid, Spain
}

Received 5 September 2007 / Accepted 3 December 2007

\begin{abstract}
Context. Eris is the largest dwarf planet currently known in the solar system. Knowledge about its physical parameters is necessary to interpret the characteristics of these kinds of bodies.

Aims. The goal of this work is to study Eris' short-term and long-term variability in order to determine the amplitude of the lightcurve, which can be linked to the degree of elongation of the body or to the degree of albedo heterogeneity on the surface of the dwarf planet. In addition, the rotation period can be determined.

Methods. CCD photometric observations of the trans Neptunian object Eris in $R$ band on 16 nights spanning two years were carried out using the $1.5 \mathrm{~m}$ telescope at Sierra Nevada Observatory (OSN), the $2.5 \mathrm{~m}$ Isaac Newton Telescope (INT) telescope at the Roque de los Muchachos Observatory, and the $2.2 \mathrm{~m}$ Telescope at Calar Alto Observatory.

Results. The time-series analysis leads to indications of a short-term variability whose nature is not clear. It could be real or a result of data-reduction artifacts, such as contamination by close, faint-background stars. The most significant periodicities are $14 \mathrm{~h}$ or its double, but other possibilities cannot be ruled out, like a $32 \mathrm{~h}$ weaker peak in the periodogram. As for the amplitude of the lightcurve, we get a peak-to-peak variability of $0.01 \pm 0.01 \mathrm{mag}$. The study of the long-term variability indicates that a long rotation period cannot be rejected, but the amplitude would be smaller than 0.06 mag. These results are compatible with a nearly spherical body that has a homogeneous surface.
\end{abstract}

Key words. Kuiper Belt - minor planets, asteroids - planets and satellites: individual: Eris

\section{Introduction}

The object (136199) Eris, formerly called $2003 \mathrm{UB}_{313}$, is the largest dwarf planet currently known in the solar system. Brown et al. (2006b), using the Hubble Space Telescope directly measured the size of Eris and determine a diameter of $(2400 \pm$ $100) \mathrm{km}$ or a size $5 \%$ larger than Pluto. Bertoldi et al. (2006) measured the thermal emission of Eris at a wavelength of $1.2 \mathrm{~mm}$, and combined their observations with the measured optical brightness, leading to a diameter of $(3000 \pm 300) \mathrm{km}$. On the other hand, Stansberry et al. (2007) using the Spitzer telescope determined a diameter of $(2657 \pm 200) \mathrm{Km}$. Eris is a trans Neptunian object (TNO), orbiting the Sun in the scattered disc with a semi-major axis of $67.668 \mathrm{AU}$, an eccentricity of 0.441 , and an inclination of 44.187 degrees.

The visual albedo of Eris was estimated as $(86 \pm 7) \%$ by Brown et al. (2006a) and ( $70 \pm 20) \%$ by Stansberry et al. (2007). Eris is less red than Pluto (Brown et al. 2005), possibly because Pluto's darkest regions appear to be a major source of the red color (Young et al. 1999). Although Eris is 20\% brighter than Pluto in absolute terms, it is only 5\% larger, so the albedo of Eris is even higher than that of Pluto.

* Table 2 is only available in electronic form at the CDS via anonymous ftp to cdsarc.u-strasbg.fr (130.79.128.5) or via http://cdsweb.u-strasbg.fr/cgi-bin/qcat?J/A+A/479/877
Adaptive optics observations of Eris at the Keck telescopes in Hawaii revealed a moon, called Dysnomia, in orbit around Eris (Brown et al. 2006b). The discovery of the satellite presented the opportunity to measure the mass of Eris. Brown \& Schaller (2007) used the period and semi-major axis of the orbit of Dysnomia to determine the mass for the Eris-Dysnomia system as $\left(1.66 \times 10^{22} \pm 0.02\right) \mathrm{kg}$, or $1.27 \pm 0.02$ of the mass of Pluto.

The infrared-reflectance spectrum of Eris is dominated by absorption due to frozen methane (Brown et al. 2005) and traces of $\mathrm{N}_{2}$ (Licandro et al. 2006), similar to the surfaces of Pluto, Charon, and Triton. Methane ice appears to be more evenly distributed over the surface of Eris than it is on Pluto, potentially masking underlying areas of red color. Unlike the somewhat reddish Pluto and Triton, however, Eris appears almost grey. Pluto's reddish color is believed to be due to deposits of tholins on its surface, and where these deposits darken the surface, the lower albedo leads to higher temperatures and the evaporation of methane deposits. In contrast, Eris is far enough away from the Sun that methane can condense onto its surface even where the albedo is low. The condensation of methane uniformly over the surface most likely reduces any albedo contrasts and covers up any deposits of red tholins. Eris and (136472) $2005 \mathrm{FY}_{9}$ both have abundant methane ice on their surfaces and so are expected to have high albedos. At Eris $38 \mathrm{AU}$ perihelion equilibrium 
Table 1. Dates, geometric, and photometric data of the observations.

\begin{tabular}{lcccccccc}
\hline \hline Teles. & Date & \# Images & $r_{\mathrm{h}}(\mathrm{AU})$ & $\Delta(\mathrm{AU})$ & $\alpha(\mathrm{deg})$ & Landolt & Mag $(R)_{\text {red }}$ & Absolute $(R)$ Mag. \\
\hline OSN & 09 Sep. 2005 & 14 & 96.905 & 96.099 & 0.35 & & & \\
OSN & 11 Sep. 2005 & 14 & 96.905 & 96.081 & 0.34 & & & \\
OSN & 03 Oct. 2005 & 40 & 96.902 & 95.950 & 0.18 & PG 2213006 & $18.28 \pm 0.01$ & $-1.55 \pm 0.01$ \\
OSN & 04 Oct. 2005 & 96 & 96.901 & 95.947 & 0.18 & PG 2213006 & $18.25 \pm 0.04$ & $-1.59 \pm 0.04$ \\
OSN & 05 Oct. 2005 & 102 & 96.901 & 95.945 & 0.17 & PG 2213006 & $18.21 \pm 0.02$ & $-1.62 \pm 0.02$ \\
OSN & 06 Oct. 2005 & 34 & 96.901 & 95.942 & 0.16 & & & \\
OSN & 11 Oct. 2006 & 7 & 96.850 & 95.885 & 0.15 & PG 2213006 & $18.36 \pm 0.06$ & $-1.47 \pm 0.06$ \\
OSN & 12 Oct. 2006 & 43 & 96.850 & 95.884 & 0.14 & PG 2213006 & $18.41 \pm 0.02$ & $-1.42 \pm 0.02$ \\
OSN & 13 Oct. 2006 & 25 & 96.850 & 95.884 & 0.14 & PG 2213006 & $18.40 \pm 0.02$ & $-1.43 \pm 0.02$ \\
OSN & 14 Oct. 2006 & 30 & 96.850 & 95.884 & 0.14 & PG 2213006 & $18.44 \pm 0.03$ & $-1.41 \pm 0.03$ \\
OSN & 15 Oct. 2006 & 3 & 96.850 & 95.884 & 0.14 & & & \\
INT & 22 Oct. 2006 & 8 & 96.849 & 95.893 & 0.16 & & & \\
INT & 23 Oct. 2006 & 4 & 96.849 & 95.896 & 0.17 & & & \\
INT & 26 Oct. 2006 & 5 & 96.848 & 95.905 & 0.18 & & & $-1.44 \pm 0.02$ \\
CAHA & 11 Jan. 2007 & 1 & 96.838 & 96.848 & 0.58 & PG 95 142 & $18.41 \pm 0.02$ & $-1.48 \pm 0.02$ \\
CAHA & 15 Jan. 2007 & 1 & 96.837 & 96.916 & 0.58 & PG 95 142 & $18.37 \pm 0.02$ & \\
\hline
\end{tabular}

temperature of $43 \mathrm{~K}$, the vapor pressure over a solid nitrogen surface would be $30 \mathrm{~Pa}$, while the pressure over a solid methane surface would be $0.2 \mathrm{~Pa}$. At the current distance of $97 \mathrm{AU}$ and at an equilibrium temperature of $27 \mathrm{~K}$, these pressures drop by 6 and 8 orders of magnitude, respectively (Brown et al. 2006b). Any nitrogen or methane atmosphere that exists at perihelion should be essentially completely frozen to the surface by aphelion.

We now have a handful of $1000 \mathrm{~km}$-sized bodies (Pluto, Eris, Quaoar, Varuna, Sedna, $2005 \mathrm{FY}_{9}, 2003 \mathrm{EL}_{61}, 2002 \mathrm{TC}_{302}$ ) whose photometric properties can be compared to each other and to smaller TNOs. While once Pluto appeared unique in the outer solar system in terms of size- and satellite-formation mechanism, it now appears to be one of a family of similar-sized objects with perhaps similar collisional histories and a range of satellite outcomes. The presence of small but discernible photometric variations on $2005 \mathrm{FY}_{9}$ (Ortiz et al. 2007) and the evidence for a small surface coverage of warm dark material suggests that $2005 \mathrm{FY}_{9}$ could have undergone partial atmospheric freezeout. But at the temperature of $2005 \mathrm{FY}_{9}$ the process is not as complete as on Eris. The process of atmospheric freezeout, which has long been debated for Pluto (Stern et al. 1988; Stansberry \& Yelle 1999; Elliot et al. 2003), can now be studied in a growing population of methane-rich objects at a wide range of distances and temperatures in the outer solar system.

With the goal of determining the spin period of Eris as well as the range of brightness variation, we performed an extensive broad-band CCD-photometry observational campaign. One of the main purposes of our campaign was to obtain the lightcurve variation that could be associated with the body deformation, any feature in the surface or spin axis direction.

\section{Observations and data reduction}

We observed at the Instituto de Astrofísica de Andalucía $1.5 \mathrm{~m}$ telescope at Sierra Nevada Observatory (OSN), Granada, Spain, from the $2.5 \mathrm{~m}$ Isaac Newton Telescope (INT) at Roque de Los Muchachos Observatory, La Palma, Spain, and also at the $2.2 \mathrm{~m}$ Telescope at Calar Alto Observatory (CAHA), Spain. We used data from 16 nights in 5 different observing runs. We first observed in September 2005 and conducted the last observation in January 2007. The observing logs are shown in Table 1. The typical seeing during the observations ranged from 1.0 to 2.0 arcsec, with median around 1.4 arcsec. The $1.5 \mathrm{~m}$ telescope observations used a $2 \mathrm{k} \times 2 \mathrm{k}$ CCD with a total field of view of $7.8 \times$ 7.8 arcmin. However, we used always $2 \times 2$ binning, giving a $0.46 \mathrm{arcsec} / \mathrm{pixel}$ scale. This scale was enough to have good point spread function sampling even for the best seeing cases. We obtained the $2.5 \mathrm{~m}$ INT telescope observations with the Wide Field Camera (WFC) instrument. The WFC consists of 4 thinned EEV $2 \mathrm{k} \times 4 \mathrm{k}$ CCDs. The CCDs have a pixel size of 13.5 microns corresponding to $0.33^{\prime \prime} /$ pixel. We performed the Calar Alto observations with the Calar Alto Faint Object Spectrograph (CAFOS) equipped with a $2048 \times 2048$ pixel CCD giving an imaging scale of $0.53^{\prime \prime} /$ pix.

Because the object's drift rate was small (approximately $1 \operatorname{arcsec} / \mathrm{h}$ ), we used a short enough exposure time in order not to get elongated images of either the object or the field stars (depending on whether the telescope is tracked at sidereal or nonsidereal rate respectively), but long enough to get a high signal to noise ratio for the object. An exposure time of $400 \mathrm{~s}$ was short enough to avoid noticeable trailing under the best foreseeable seeing conditions, but long enough so that the sky background was the dominating noise source. We observed in the Johnson Cousins $R$ filter.

We bias subtracted the images in the standard way, and flatfielded using a daily master flatfield frame (obtained as the median of a large set of dithered twilight images of the sky at blank fields). We did not use cosmic ray removal algorithms, and we rejected the images in which a cosmic ray hit or stars were close to the object. Few observations were adversely affected by cosmic-ray hits within the flux aperture. We do not include these observations in our results. We used Daophot routines to carry out relative photometry of six field stars. The synthetic aperture used was typically 3 to 5 arcsec in diameter (the smallest possible to get the highest signal to noise). We took care not to introduce spurious signals of faint background stars or galaxies in the aperture. Because of the small angular motion of the dwarf planet, we were able to use the same field stars within each observing run. The typical error bars of the individual $400 \mathrm{~s}$ integrations were $\sim 0.01 \mathrm{mag}$. A sample of a combination of images with Eris and the reference stars is shown in Fig. 1.

We inspected the time-resolved observations for periodicities with the Lomb technique (Lomb 1976) as implemented by Press et al. (1992). We also verified the results with several other time-series analysis techniques (such as PDM, and the 


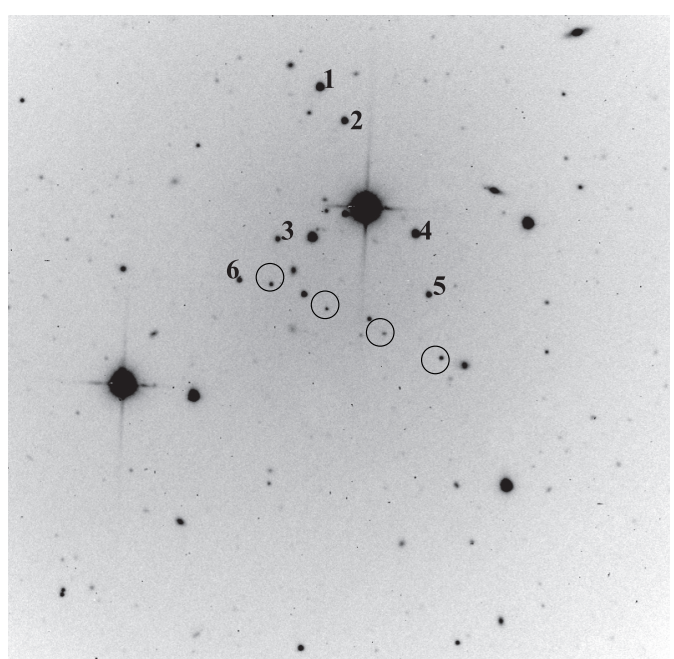

Fig. 1. A $7 \times 7$ arcmin window of a representative image of Eris taken on October 12, 13, 14 and 15, 2006 at OSN. Circles indicate where Eris was during the 4 night campaign, and numbers indicates the reference stars used.

Harris et al. 1989, method). The reference stars were also inspected for short term variability. We can thus be confident that we introduced no error by the choice of reference stars.

Since we observed the object in more than one campaign, the reference stars were not the same in different runs. To apply time-series analysis to different data sets of a same object separated by several weeks or months, we scaled the data to the mean flux of each different run so that they gave the same mean value. Since an absolute calibration would not be accurate enough and even if it were, the phase effect would introduce shifts to the data of each run, we preferred to normalize each run to one and apply the time-series analysis to the data combined in this way. We are implicitly assuming that the rotation period would be shorter than the typical 5-day observing runs. Nevertheless in our longest, 5-night run we checked that there was no continuous brightening or dimming trend in the dataset, which might be indicative of a long rotation period.

\section{Results}

We analyzed more than 400 images, amounting a total observing time of more than $47 \mathrm{~h}$ of Eris. The photometry results from those images cannot be shown on a printed table because of the huge length. The data will be available as supplementary on-line material (Table 2).

The Lomb periodogram for the relative photometry (Fig. 2) shows several peaks. The peak with the highest spectral power (with a confidence level well above the $99.99 \%$ ) corresponds to a periodicity of $13.7 \mathrm{~h}$ ( 1.75 cycles/day). The periodogram shows other peaks of weaker spectral power around the $32 \mathrm{~h}$ range. By studying the data with a different time-series analysis techniques such as PDM, one gets the preferred period at $\sim 14 \mathrm{~h}$. We obtained the preferred period at $28.03 \mathrm{~h}$ with the Harris method with 2-harmonic. This is close to double the $13.7 \mathrm{~h}$ period obtained with the Lomb periodogram and therefore implies a double-peaked lightcurve.

Visual inspection of the rotational phase curves for the above mentioned periodicities seems to slightly favor the $13.7 \mathrm{~h}$, as can be seen in Fig. 3. According to the rotational phase curves, the

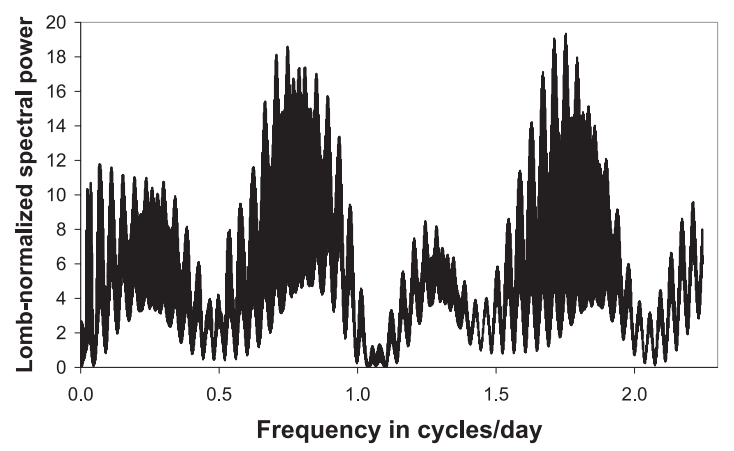

Fig. 2. Lomb periodogram for the Eris relative photometry showing several peaks (in cycles per day). The highest peaks are at 1.75 cycles/day $(P=13.7 \mathrm{~h})$ and 0.72 cycles/day $(P=32.1 \mathrm{~h})$.
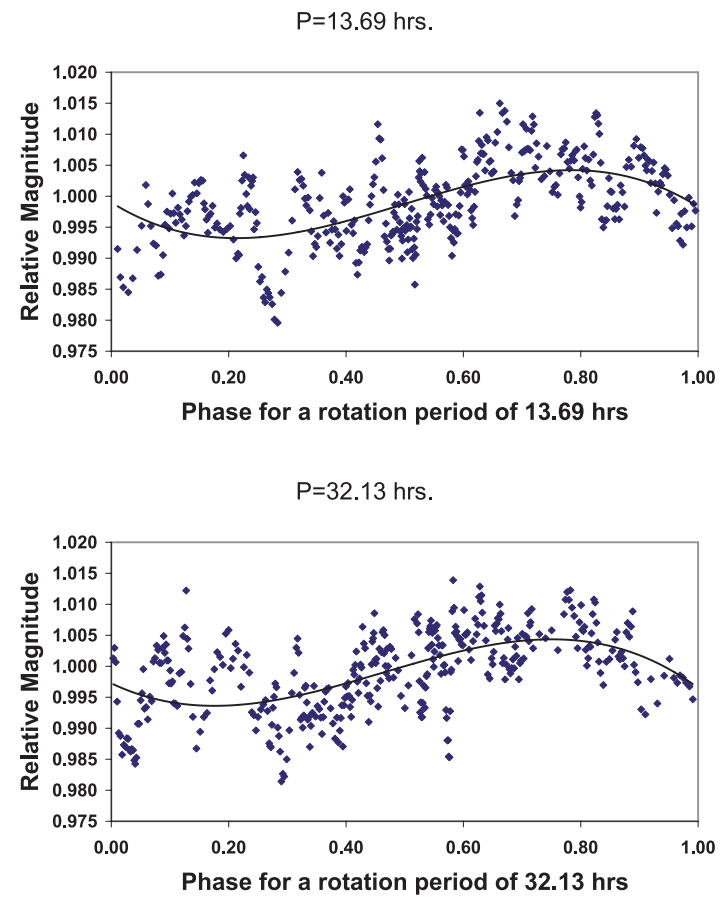

Fig. 3. Lightcurves (relative magnitudes vs. rotational phase) for the two possible periods. Top: for a $13.7 \mathrm{~h}$ period. Bottom: for a $32.1 \mathrm{~h}$ period.

spin period of this dwarf planet could be around $14 \mathrm{~h}$ or $28 \mathrm{~h}$. For all of these periodicities, the peak-to-peak amplitude of the lightcurve is $0.01 \pm 0.01 \mathrm{mag}$ (from sinusoidal fits to the data). These results on amplitude and rotation period are compatible with Carraro et al. (2006) who carried out time-series photometry of Eris and estimate a period of about $30 \mathrm{~h}$. Carraro et al. (2006) could not determine a precise rotation period because of fewer data points. We believe that the periodicity we derive may be real, but because the amplitude is small, it is possible that the data contained small systematic errors like contamination from faint background stars. If the object is passing close to a star 5 mag dimmer than Eris, it could introduce a signal greater than the photometric error and could cause spurious results.

During some of the nights, we observed Landolt star-fields to obtain the reduced magnitude of Eris. The reduced and absolute magnitudes are shown in Table 1 . We obtained the reduced and absolute magnitude for 3 different observational campaigns in October 2005 (marked as IAA2005), October 2006 (IAA2006) 


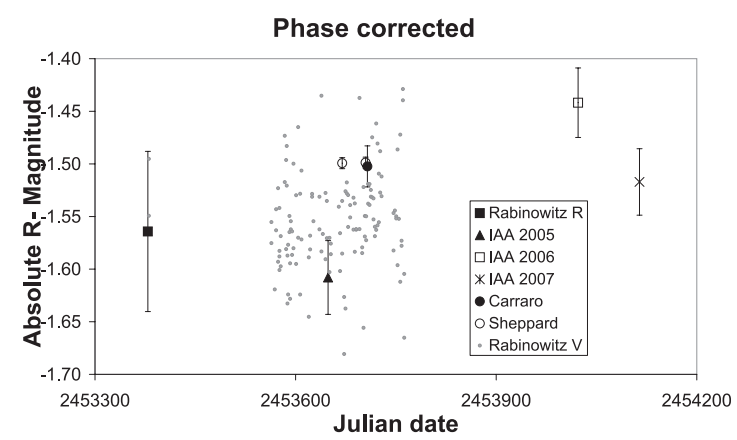

Fig. 4. Absolute magnitude, obtained in this work, and compared with that of other authors. Plotted are the mean data from Rabinowitz et al. (2006) in $R$ filter (filled square) and $V$ filter (small dots, corrected with the color index $V-R=0.45$ as explained in the text), Carraro et al. (2006) (filled circle), Sheppard (2007) (white circles) and our work of 2005 in filled triangle, 2006 (white square) and 2007 (black cross). All data are phase corrected using the phase coefficient of $0.09 \mathrm{mag} / \mathrm{deg}$ (Sheppard 2007).

and January 2007 (IAA2007). We compared these results after a zero-phase correction using a phase coefficient of $0.09 \mathrm{mag} / \mathrm{deg}$ (Sheppard 2007). They are consistent with those obtained by other authors (Rabinowitz et al. 2006; Sheppard 2007; Carraro et al. 2006) within 0.06 mag. They are all plotted together in Fig. 4. This means that we cannot reject a long term period of 0.06 mag peak-to-peak variation, although absolute calibration errors are most likely the cause of the spread.

The first noticeable fact in this curve is the small decrease in the brightness of Eris during the 3 years of observations. Another important fact is that our data of October 2005 (marked as IAA2005) seem to be out of the curve. we checked this point independently with two different reduction procedures and obtained the same results. To compare and have more confidence in our results, we decided to plot all the data that are accessible in the literature. The work of Rabinowitz et al. (2006) has the most extensive results both, in time and quantity. Figure 4 shows all the data they published in $V$ and $R$ filter, phase corrected. The observations in $V$ filter show a minimum around October 2005. If a color index $V-R=0.45$ (Brown et al. 2005) is applied to their observations, the IAA2005 point will fit with that of Rabinowitz's $V$-filter curve. We are unsure whether this minimum is real or not.

On the other hand, it needs to be mentioned that each of the authors cited in Fig. 4 obtained a large scatter in their data for each night. For example, Rabinowitz et al. (2006) obtained a difference in the absolute magnitude as large as 0.2 mag in both filters. To explain a possible 3 -year decrease in the brightness, Jewitt \& Luu (2004) presented the possibility that crystalline ice can be turned into amorphous ice in TNOs by ultraviolet light and cosmic radiation. In this process, the surface of the body will be redder and darker, similar to the resurfacing of Pluto.

\section{Discussion and conclusions}

In this paper, we presented a large photometry data set for Eris. Contrary to Pluto and similarly to $2005 \mathrm{FY}_{9}$, Eris exhibits small amplitude lightcurve variations and perhaps a shorter, single peaked, variation of nearly $14 \mathrm{~h}$ or $28 \mathrm{~h}$ (if double peaked), or the $32 \mathrm{~h}$ period that can not be ruled out. However, there are many aliases in the periodogram with similar spectral power and therefore it is difficult to determine the exact rotation period. Besides, the existence of systematic errors or biases is difficult to rule out because of the small variability in the lightcurve amplitude.

If real, the brightness changes would likely be due to the combined effect of rotation, irregular shape and/or albedo markings on its surface. Double-peaked lightcurves are usually attributed to the rotation of an irregularly-shaped body while single-peaked curves are attributed to albedo variations on the surface. It is not possible to determine if the variations in Eris lightcurve are due to albedo differences or elongated shape, but we prefer the albedo option due to the size of the object. So far, the only method to reliably distinguish shape-induced from albedo-induced brightness variations is simultaneous observations in the visible and in the thermal. If Eris were a spherical body (its large size and slow rotation suggests a nearly-spherical body), the photometric range of the lightcurve would imply that the maximum and minimum values of the disk-integrated albedo would differ by $2 \%$ at most. This could be caused by the presence of a large area with slightly different albedo than the other hemisphere, or by a different configuration of bright/dark spots. At the 97 AU distance of Eris, however, even dark regions should be sufficiently cold that methane freezes out of the atmosphere. Thus, methane ices would cover the entire body, lowering albedo contrasts and hiding the red tholins. On the other hand, cryovolcanism could cover the surface of the body, leading to the same phenomenology of a homogenous surface.

The process of atmospheric freezeout (the periodic ice resurfacing by sublimation near perihelion and recondensation of volatiles farther from the Sun) could be a plausible explanation for the low contrast in the lightcurve. Depending on the details of such resurfacing mechanism and atmospheric circulation, it would be a surprise to discover significant differences between different areas on the surface of Eris. Other ice resurfacing scenarios are possible.

Licandro et al. (2006), compared the spectra of Eris, $2005 \mathrm{FY}_{9}$, and Pluto around the 0.73 and $0.89 \mu \mathrm{m}$ spectral bands and concluded that the visible spectrum of Eris is not as red as the spectra of Pluto and $2005 \mathrm{FY}_{9}$, thus complex organics should be less abundant on the surface of Eris than on the surfaces of Pluto and $2005 \mathrm{FY}_{9}$. The object $2005 \mathrm{FY}_{9}$, which also has a surface covered with methane frost (Licandro et al. 2006) and is currently at a distance of $52 \mathrm{AU}$, should have a surface temperature approximately midway between Pluto and Eris ( 97 UA, see Table 1). The presence of small, but detectable photometric variations on $2005 \mathrm{FY}_{9}$ (Ortiz et al. 2007) and the evidence for a small surface coverage of warm dark material suggests that $2005 \mathrm{FY}_{9}$ could have undergone partial atmospheric freezeout, but that at the temperature of $2005 \mathrm{FY}_{9}$ the process is not as complete as on Eris.

Finally, from our data we can not detect the presence of the satellite Dysnomia. In the work of Brown \& Schaller (2007) two sets of orbital parameters, both of them with high orbital inclination and an orbital period of 15.77 days, are presented. In this context it is difficult to observe an eclipse in the time-period of 2005-2007. Besides, a possible short-term variability produced by Dysnomia would be far below one percent the total flux, which implies that it would be undetectable in our photometry.

Acknowledgements. We are grateful to the Sierra Nevada Observatory, Calar Alto Observatory and INT Observatory staffs. This research was partially based on data obtained at the Observatorio de Sierra Nevada which is operated by the Instituto de Astrofísica de Andalucía, CSIC. Based on observations collected at the Centro Astronómico Hispano Alemán (CAHA) at Calar Alto, operated jointly by the Max- Planck Institut für Astronomie and the Instituto de 
Astrofísica de Andalucía (CSIC). RD acknowledges financial support from the MEC (contract Juan de la Cierva). This work was supported by contracts AYA2004-03250 and AYA2005-07808-C03-01. European FEDER funds for these contracts are also acknowledged. P.J.G. acknowledges financial support from the MEC (contract Ramón y Cajal).

\section{References}

Bertoldi, F., Altenhoff, W., Weiss, A., Menten, K. M., \& Thum, C. 2006, Nature, 439, 563

Brown, M. E., \& Schaller, E. L. 2007, Science, 316, 1585

Brown, M. E., Trujillo, C. A., \& Rabinowitz, D. L. 2005, ApJ, 635, L97

Brown, M. E., Schaller, E. L., Roe, H. G., Rabinowitz, D. L., \& Trujillo, C. A. 2006a, ApJ, 643, L61

Brown, M. E., van Dam, M. A., Bouchez, A. H., et al. 2006b, ApJ, 639, L43

Carraro, G., Maris, M., Bertin, D., \& Parisi, M. G. 2006, A\&A, 460, L39

Elliot, J. L., Ates, A., Babcock, B. A., et al. 2003, Nature, 424, 165
Harris, A. W., Young, J. W., Bowell, E., et al. 1989, Icarus, 77, 171

Jewitt, D. C., \& Luu, J. 2004, Nature, 432, 731

Licandro, J., Grundy, W. M., Pinilla-Alonso, N., \& Leisy, P. 2006, A\&A, 458, L5

Lomb, N. R. 1976, Ap\&SS, 39, 447

Ortiz, J. L., Santos Sanz, P., Gutiérrez, P. J., Duffard, R., \& Aceituno, F. J. 2007, A\&A, 468, L13

Press, W. H., Teukolsky, S. A., Vetterling, W. T., \& Flannery, B. P. 1992, Numerical recipes in FORTRAN. The art of scientific computing (Cambridge: University Press), 2nd edn.

Rabinowitz, D. L., Barkume, K., Brown, M. E., et al. 2006, ApJ, 639, 1238

Sheppard, S. S. 2007, AJ, 134, 787

Stansberry, J. A., \& Yelle, R. V. 1999, Icarus, 141, 299

Stansberry, J., Grundy, W., Brown, M., et al. 2007, ArXiv Astrophysics e-prints

Stern, S. A., Trafton, L. M., \& Gladstone, G. R. 1988, Icarus, 75, 485

Young, E. F., Galdamez, K., Buie, M. W., Binzel, R. P., \& Tholen, D. J. 1999, AJ, 117, 1063 